\title{
Joint Image Formation and Anisotropy Characterization in Wide-Angle SAR
}

\author{
Kush R. Varshney $^{a}$, Müjdat Çetin ${ }^{a, b}$, John W. Fisher III $^{c}$ and Alan S. Willsky ${ }^{a, c}$ \\ ${ }^{a}$ Laboratory for Information and Decision Systems, \\ Massachusetts Institute of Technology, Cambridge, MA 02139, USA; \\ ${ }^{b}$ Faculty of Engineering and Natural Sciences, \\ Sabancı University, Orhanl, Tuzla 34956 İstanbul, Turkey; \\ ${ }^{c}$ Computer Science and Artificial Intelligence Laboratory, \\ Massachusetts Institute of Technology, Cambridge, MA 02139, USA
}

\begin{abstract}
We consider the problem of jointly forming images and characterizing anisotropy from wide-angle synthetic aperture radar (SAR) measurements. Conventional SAR image formation techniques assume isotropic scattering, which is not valid with wide-angle apertures. We present a method based on a sparse representation of aspect-dependent scattering with an overcomplete basis composed of basis vectors with varying levels of angular persistence. Solved as an inverse problem, the result is a complex-valued, aspect-dependent response for each spatial location in a scene. Our non-parametric approach does not suffer from reduced cross-range resolution inherent in subaperture methods and considers all point scatterers in a scene jointly. The choice of the overcomplete basis set incorporates prior knowledge of aspect-dependent scattering, but the method is flexible enough to admit solutions that may not match a family of parametric functions. We enforce sparsity through regularization based on the $\ell_{k}$-norm, $k<1$. This formulation leads to an optimization problem that is solved through a robust quasi-Newton method. We also develop a graph-structured interpretation of the overcomplete basis leading towards approximate algorithms using guided depth-first search with appropriate stopping conditions and search heuristics. We present experimental results on synthetic scenes and the backhoe public release dataset.
\end{abstract}

Keywords: synthetic aperture radar, wide-angle imaging, anisotropy, sparse signal representation, image formation, inverse problems

\section{INTRODUCTION}

Wide-angle synthetic aperture radar (SAR) imaging has come to the fore recently due to advances in navigation and avionics technologies that permit the synthesis of very long apertures. In principle, wide-angle measurements allow for the formation of images finely resolved in the cross-range direction. However, conventional image formation techniques are not adequate for dealing with data collected over wide-angle apertures for a number of reasons. One issue, and the focus of this paper, that arises with wide-angle apertures is that dependence of scattering behavior on aspect angle, termed anisotropy, becomes prominent because objects are viewed from different sides rather than from nearly the same point of view. This is in opposition to narrow-angle imaging, where it is a fairly reasonable assumption that scattering amplitude is constant over the aperture. In conventional image formation techniques, the failure to model angle dependence results in an averaging over that variable, leading to inaccurate scattering estimates. In addition, the anisotropy level of scatterers is not characterized. Yet, anisotropy characterization may be used as a feature for automatic target recognition and for improved image formation.

The problem of detecting, estimating, and modeling aspect-dependent scattering behavior has received attention lately. Anisotropy characterization methods may be broadly categorized into those that operate in the phase history domain, employing parameterizations for angle-dependent scattering, and those that operate in

Further author information: Send correspondence to K.R.V.: E-mail: krv@mit.edu, Telephone: 1-617-253-7220

Algorithms for Synthetic Aperture Radar Imagery XIII, edited by Edmund G. Zelnio, Frederick D. Garber, Proc. of SPIE Vol. 6237, 62370D, (2006) · 0277-786X/06/\$15 · doi: 10.1117/12.663974 
the image domain. The general parametric approach is to posit a parametric model for angle dependent scattering, often motivated by electromagnetic theory, and estimate the model parameters, leading to a well-defined estimation problem. ${ }^{1-4}$ Image-domain methods use a multiaperture approach for characterizing anisotropy. ${ }^{5-11}$ Subaperture images are formed, either conventionally or using an enhanced image formation technique, from segments of the measurements divided in aspect angle. The sequence of subaperture images then gives an indication of the persistence of the scatterers in the scene. It should be noted that the subaperture images have poorer cross-range resolution than an image formed from the full aperture would. Also, subapertures are of fixed angular extent; consequently, any subaperture analysis is limited in its ability to characterize anisotropy persistence. Parametric methods and image-domain methods have been shown to work well in different situations. Notably, the parametric models incorporate much prior information about expected scattering behavior. Also, the estimated parameters have physical significance, e.g. a parameter corresponding to the physical length of the scattering mechanism. The image-domain methods are robust, easy to reason about conceptually, and can be applied to already formed images.

The parametric model formulation of the anisotropy characterization problem is of course predicated on the correct modeling of natural phenomena. However, parametric models often do not hold in wide-angle imaging scenarios. ${ }^{10}$ Within the image-domain methods, a subaperture pyramid framework with overlapping subapertures of various angular extents moves towards allowing a continuum of aspect angle extents, but is still limited to full-, half-, quarter-, $\cdots$-, apertures. ${ }^{12}$ Also, in most techniques, the characterization of anisotropy in different spatial locations (different pixels) is done independently.

In this paper, we consider an inverse problem formulation utilizing an overcomplete basis and sparsifying regularization for joint image formation and anisotropy characterization in wide-angle SAR. Sparsifying regularization has been applied to inverse problems including acoustic source localization ${ }^{13}$ and isotropic SAR imaging, ${ }^{14}$ but has not previously been applied to the SAR anisotropy characterization problem. While still taking advantage of prior information, this method is flexible enough to admit solutions that are not from a prespecified parametric family. It jointly treats spatial locations and suffers no reduction in cross-range resolution. We also develop a graph-structured interpretation of our overcomplete basis leading towards novel approximate algorithms to solve the inverse problem. These algorithms, having reduced memory requirements, may well find application in a wide variety of sparse signal representation settings beyond the specific problem of anisotropy in SAR.

Sec. 2 describes our framework for bringing the SAR image formation and anisotropy characterization application together with the inverse problem-sparsity mathematical formalism. Specifically, an overcomplete expansion of the point-scattering observation model is proposed, along with a discussion on the choice of vectors for the expansion. In Sec. 3, we build upon the framework of the previous section, describing methods of solving the inverse problem while imposing sparsity. A quasi-Newton method ${ }^{14}$ and greedy graph-structured algorithms are applied to the problem. In Sec. 4, examples with synthetic data, with a scene composed of realistic canonical scatterers, and a scene containing the backhoe loader of the Backhoe Data Dome ${ }^{15}$ are given. We provide some discussion of the results in the concluding section.

\section{OVERCOMPLETE BASIS FORMULATION FOR ANISOTROPY CHARACTERIZATION AND IMAGE FORMATION}

In this section, we describe a formulation of the anisotropy characterization problem which differs from the subaperture and parametric formulations mentioned in Sec. 1. The problem is approached by constructing an overcomplete basis and appropriately using the phase history measurements. An overcomplete basis, also known as an overcomplete dictionary, is more than a basis, i.e. a collection containing more vectors than necessary to span the space and hence, a linearly dependent set. The idea is to expand the aspect-dependent scattering function $s(\theta)$ at each spatial location as a superposition of basis vectors and then determine coefficients for those vectors. The first part of the section leaves the overcomplete basis fully general; the section concludes with a consideration of specific basis choices.

\subsection{Anisotropy Characterization Inverse Problem}

In two-dimensional imaging, the goal is to determine the complex-valued scattering function of a ground patch $s(x, y)$, where $x$ and $y$ are coordinates with origin at the center of that ground patch in the range and cross-range 
directions, respectively. However, due to anisotropy, scattering depends on aspect angle $\theta$, with the scattering function taking the form $s(x, y, \theta)$. In our work, we aim to jointly characterize anisotropy and form images by determining this function $s(x, y, \theta)$. The starting point for our overcomplete expansion is the phase history observation model for point scattering centers with anisotropy, given below.

$$
r(f, \theta)=\sum_{p=1}^{P} s\left(x_{p}, y_{p}, \theta\right) \exp \left\{-j \frac{4 \pi f}{c}\left(x_{p} \cos \theta+y_{p} \sin \theta\right)\right\},
$$

where $c$ is the speed of propagation and $f$ is the frequency of the radar measurements. For a single spatial location $p$, we expand the aspect-dependent scattering as follows:

$$
s\left(x_{p}, y_{p}, \theta\right)=\sum_{m=1}^{M} a_{p, m} b_{m}(\theta)
$$

yielding the following overall $M \cdot P$ vector basis expansion:

$$
r(f, \theta)=\sum_{p=1}^{P} \sum_{m=1}^{M} a_{p, m} b_{m}(\theta) \exp \left\{-j \frac{4 \pi f}{c}\left(x_{p} \cos \theta+y_{p} \sin \theta\right)\right\} .
$$

Isotropic scattering is a special case of the above expression with $M=1$ and $b_{1}(\theta)$ constant.

Assuming that the phase history measurements are at $K$ discrete frequencies and $N$ discrete aspect angles, let us define length $N$ vectors $\mathbf{r}_{k}=r\left(f_{k}, \theta\right), \mathbf{b}_{m}=b_{m}(\theta)$, and $\varepsilon_{k, p}=\exp \left\{-j \frac{4 \pi f_{k}}{c}\left(x_{p} \cos \theta+y_{p} \sin \theta\right)\right\}$. Then, taking $\phi_{k, p, m}=\mathbf{b}_{m} \varepsilon_{k, p}$, the basis expansion may be simply expressed as:

$$
\mathbf{r}_{k}=\sum_{p=1}^{P} \sum_{m=1}^{M} a_{p, m} \phi_{k, p, m}, k=1, \ldots, K
$$

The inverse problem is to determine the $M \cdot P$ complex-valued coefficients $a_{p, m}$ that satisfy or approximately satisfy the linear equations (1). We choose the number of basis vectors $M$ such that $M>N$, making the basis overcomplete.

Now, let us move to matrix-vector equations to simplify the discussion. The collection of all phase history measurements can be stacked as the following tall $N \cdot K$-vector $\mathbf{r}$.

$$
\mathbf{r}=\left[\begin{array}{c}
\mathbf{r}_{1} \\
\mathbf{r}_{2} \\
\vdots \\
\mathbf{r}_{K}
\end{array}\right]
$$

The set of all basis vectors at a particular frequency $f_{k}$ and spatial location $\left(x_{p}, y_{p}\right)$ can be collected into a matrix $\boldsymbol{\Phi}_{k, p}=\left[\begin{array}{llll}\phi_{k, p, 1} & \phi_{k, p, 2} & \cdots & \phi_{k, p, M}\end{array}\right]$. In the same manner, the $\mathbf{b}_{m}$ vectors can be concatenated into a matrix $\mathbf{B}=\left[\begin{array}{llll}\mathbf{b}_{1} & \mathbf{b}_{2} & \cdots & \mathbf{b}_{M}\end{array}\right]$. These two matrices are related by the expression $\boldsymbol{\Phi}_{k, p}=\mathbf{B} \bullet\left(\varepsilon_{k, p} \mathbf{1}_{M}^{T}\right)$, where $\mathbf{M}_{1} \bullet \mathbf{M}_{2}$ is the elementwise multiplication of matrices $\mathbf{M}_{1}$ and $\mathbf{M}_{2}$, and $\mathbf{1}_{M}$ is the $M$-vector of all ones. The factor $\mathbf{B}$ is subject to design in the anisotropy characterization procedure, but $\boldsymbol{\varepsilon}_{k, p}$ is fundamental to the SAR phase history measurements. The choice of $\mathbf{B}$ is discussed in the second half of this section.

Putting together all frequencies and spatial locations, the overall overcomplete basis $\boldsymbol{\Phi}$ is:

$$
\boldsymbol{\Phi}=\left[\begin{array}{cccc}
\boldsymbol{\Phi}_{1,1} & \boldsymbol{\Phi}_{1,2} & \cdots & \boldsymbol{\Phi}_{1, P} \\
\boldsymbol{\Phi}_{2,1} & \boldsymbol{\Phi}_{2,2} & \cdots & \boldsymbol{\Phi}_{2, P} \\
\vdots & \vdots & \ddots & \vdots \\
\mathbf{\Phi}_{K, 1} & \boldsymbol{\Phi}_{K, 2} & \cdots & \boldsymbol{\Phi}_{K, P}
\end{array}\right]
$$




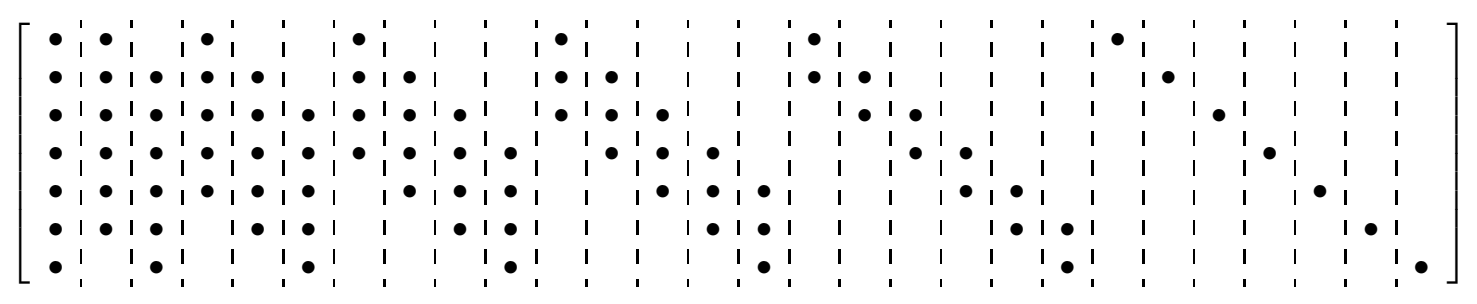

Figure 1. Illustration of matrix $\mathbf{B}$ for $N=7$.

Each column of the matrix $\boldsymbol{\Phi}$ is a basis vector of the overcomplete basis.

Defining the length $M \cdot P$ vector of coefficients as $\mathbf{a}=\left[\begin{array}{lllllll}a_{1,1} & a_{1,2} & \cdots & a_{1, M} & a_{2,1} & \cdots & a_{P, M}\end{array}\right]^{T}$, the statement $\mathbf{r}=\mathbf{\Phi} \mathbf{a}$ in matrix-vector form is completely equivalent to the summation form (1). In this form, it is readily apparent that there are $N \cdot K$ linear equations with $M \cdot P$ unknowns and $M>N$. Often in the choice of $\mathbf{B}$, $M \gg N$, so regardless of $P$ and $K, M \cdot P>N \cdot K$ and the system is an underdetermined set of linear equations. Our formulation also readily deals with the noisy case: $\mathbf{r}=\mathbf{\Phi} \mathbf{a}+\mathbf{n}$, where $\mathbf{n}$ is an additive noise term. We delay discussion of solving this inverse problem for a to Sec. 3 .

By forming this $\boldsymbol{\Phi}$ matrix, the spatial locations are treated jointly within one system of equations, capturing the combined influence of multiple scatterers on individual angle-frequency measurements. The first $M$ elements of $\mathbf{a}$ depend on the first spatial location $p=1$, elements $M+1$ to $2 M$ of a depend on the second position $p=2$, and so on. Thus, by setting up the problem in this manner, it is possible to decompose the phase history data into contributions from different point scatterers at different locations and in the process, characterize amplitude and anisotropy for each one. As a notational convenience, we define $\mathbf{a}_{p}$ to be the $M$-vector of coefficients corresponding to position $p$ and:

$$
\boldsymbol{\Phi}_{p}=\left[\begin{array}{c}
\boldsymbol{\Phi}_{1, p} \\
\boldsymbol{\Phi}_{2, p} \\
\vdots \\
\boldsymbol{\Phi}_{K, p}
\end{array}\right]
$$

to be the subset of basis vectors corresponding to position $p$. There is no requirement that all spatial locations under consideration contain a scatterer. If there is no scatterer at a particular spatial location $p$, then all of the elements of $\mathbf{a}_{p}$ should come out to be zero. It is thus possible to use a grid of pixels as the set of potential spatial locations where scatterers might exist. We now discuss the specific choice of basis vectors for the overcomplete basis $\boldsymbol{\Phi}$.

\subsection{Choice of Basis Vectors}

The overcomplete basis set is to be chosen such that its cardinality is much greater than the dimension of $\theta$ and linear combinations of very few basis vectors accurately represent plausible angle-dependent scattering behaviors. In the selection of the overcomplete basis $\mathbf{\Phi}$, we are free to choose $\mathbf{B}$; the choice of $\mathbf{B}$ is a way to incorporate prior information about angle-dependent scattering.

Methods employing subaperture analysis and parametric models expect to find contiguous intervals in $\theta$ for which there is non-zero scattering. Similarly here, basis vectors are chosen such that contiguous segments of anisotropy are represented by a single basis vector. However, our formulation allows the representation of noncontiguous segments through the combination of multiple basis vectors. The $\mathbf{b}_{m}$ are chosen to be pulses with all possible angular extents and all possible starting angles, in other words all widths and shifts. For example, if $N=7$ and the pulse shape is rectangular, then $\mathbf{b}_{1}$, the isotropic vector, is $\left[\begin{array}{lllllll}1 & 1 & 1 & 1 & 1 & 1 & 1\end{array}\right]^{T}, \mathbf{b}_{2}=\left[\begin{array}{lllllll}1 & 1 & 1 & 1 & 1 & 1 & 0\end{array}\right]^{T}$, $\mathbf{b}_{3}=\left[\begin{array}{llllll}0 & 1 & 1 & 1 & 1 & 1\end{array}\right]^{T}$, and the final pulse with the finest anisotropy $\mathbf{b}_{M}=\left[\begin{array}{lllllll}0 & 0 & 0 & 0 & 0 & 0 & 1\end{array}\right]^{T}$. The $\mathbf{b}_{m}$ have unit maximum amplitude; solving the inverse problem gives the complex amplitude coefficients $\mathbf{a}$. The full set $\mathbf{B}$ for $N=7$ is illustrated in Fig. 1. The dots represent entries that have a non-zero value and spaces without dots represent zero-valued elements. For this choice of basis vectors, $M=\frac{1}{2} N^{2}+\frac{1}{2} N$. Various pulse shapes, not just 


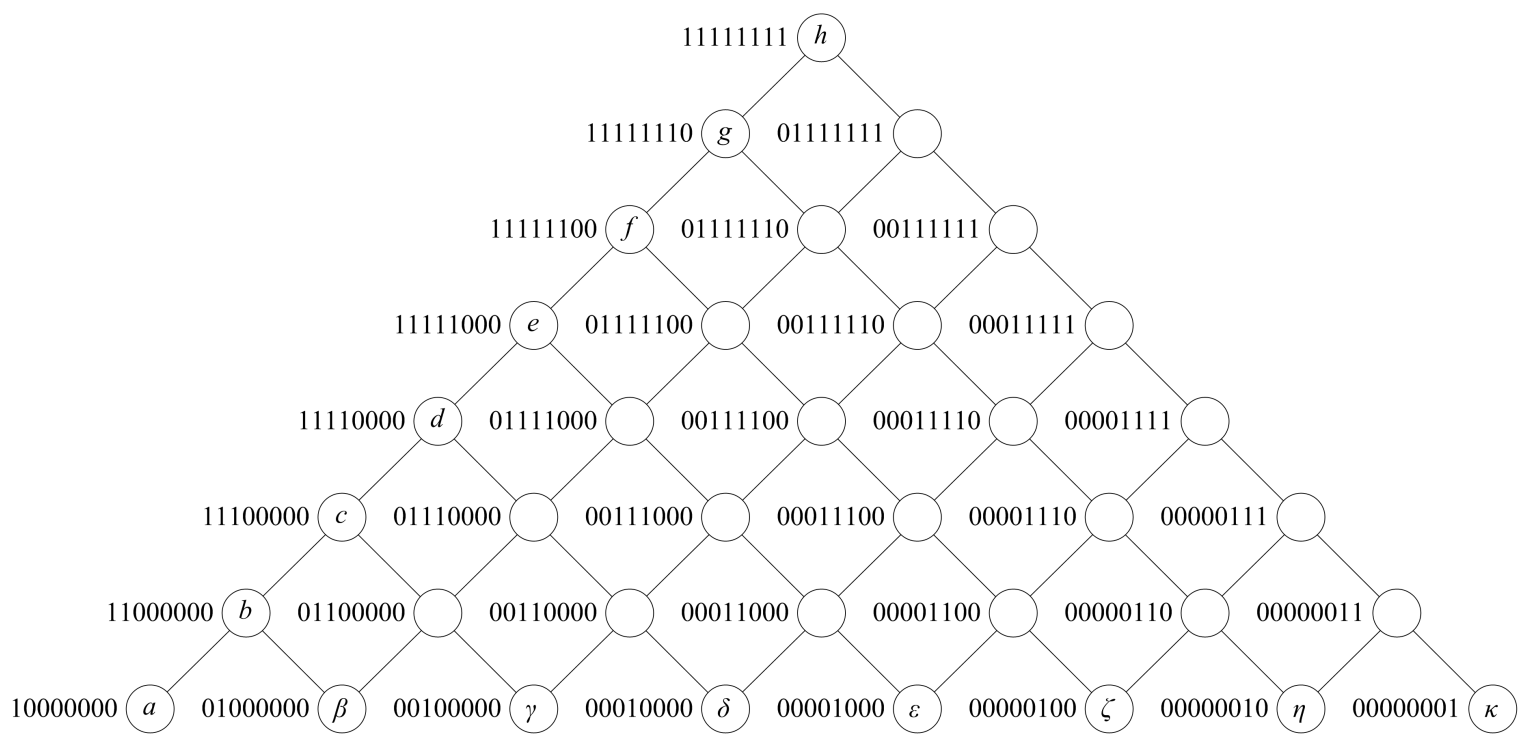

Figure 2. Graph-structured representation of B.

rectangular pulses, may be seamlessly enlisted in the overcomplete basis, e.g. triangle, raised triangle, windowed Gaussian, and Hamming pulse shapes.

The collection $\mathbf{B}$ described above has a nice, intuitive graph-structured interpretation. The vectors $\mathbf{b}_{m}$ can be arranged as nodes in a graph. The graph is given in Fig. 2 for $N=8$, with nodes labeled to the left with their corresponding $\mathbf{b}$ vectors when the pulse shape is rectangular. The labels inside the nodes may be ignored for now. The graph has $N$ levels, with the root node being the isotropic basis vector; traversing down the graph corresponds to decreasing angular extent of anisotropy. A graph of this form is referred to as an $N$-level basis graph in the remainder of this paper. This structure will be useful in the development of greedy algorithms in the next section, which discusses methods to solve the inverse problem $\mathbf{r}=\mathbf{\Phi} \mathbf{a}$.

\section{METHODS OF SOLUTION TO THE INVERSE PROBLEM}

From linear algebra, we know that the underdetermined system of linear equations (1) has no unique solution. Furthermore, the overcomplete basis $\boldsymbol{\Phi}$ is designed to allow for the representation of the phase history measurements with few basis vectors. Thus the inverse problem is a sparse signal representation problem - among the infinite number of solutions, our formulation favors those solutions that are sparse, i.e. those solutions a whose $\ell_{0}$-norm is small.

Finding the solution that minimizes $\|\mathbf{a}\|_{0}$ is a combinatorial optimization problem, but greedy approaches such as matching pursuit, and relaxations such as the $\ell_{1}$ relaxation matching pursuit have been developed. A sparsifying regularization approach incorporating a quasi-Newton optimization algorithm, originally developed for feature-enhanced SAR image formation ${ }^{14}$ but applicable to a variety of sparse signal representation problems, is an alternative method. The objective is to find the solution that minimizes the cost function $J(\mathbf{a})$, containing two terms, a data fidelity term and a sparsifying term. Specifically, the form of the cost function is:

$$
J(\mathbf{a})=\|\mathbf{r}-\mathbf{\Phi} \mathbf{a}\|_{2}^{2}+\alpha\|\mathbf{a}\|_{k}^{k}, k<1 .
$$

The $\ell_{k}$-norm with $k<1$ has a sparsifying effect. The scalar $\alpha$ is a regularization parameter that trades off data fidelity and sparsity. Details of this robust method may be found in Ref. 14.

In theory, there is no restriction on the size of the problem that the quasi-Newton method can be applied to. However, the number of columns of $\boldsymbol{\Phi}$, which is $\mathcal{O}\left(N^{2} P\right)$ for the overcomplete basis choice discussed in Sec. 2.2, 
is restrictive in terms of memory as well as computation for realistic imaging scenarios with hundreds of angle samples and spatial locations. In this section we develop a greedy algorithm with reduced memory requirements taking advantage of the graph structure described in Sec. 2.2.

In the $N$-level basis graph, the nodes represent the basis vectors in the overcomplete basis. The basis is designed such that a few or often just one basis vector per position $p^{\prime}$ is sufficient to represent the aspectdependent scattering function $s\left(x_{p^{\prime}}, y_{p^{\prime}}, \theta\right)$. Thus, the sparse signal representation problem may be reformulated as a search for a node or a few nodes on the basis graph. In addition to finding nodes, complex amplitudes must also be determined. In general, there are $P>1$ spatial locations in the problem, and consequently $P$ coexisting basis graphs. Thus, to solve the problem, there is not just one search to be done, but $P$ simultaneous searches. To be most effective, these searches should not be performed independently, but rather should interact and influence each other.

We propose a search strategy akin to guided depth-first search per basis graph, which follows a single path down from the root looking for the goal. Each step in the search is based on a heuristic. If the bottom of the graph is reached without finding the goal, then there is back-tracking also based on the heuristic. Nodes in the basis graph have two children; when progressing downwards during the search, the heuristic is used to determine whether the next step is the left or right child node. Unlike standard graph search problems, in our problem it is not obvious when to terminate the search, so we also need to specify stopping conditions.

Our search heuristic and stopping criterion is founded on solving the inverse problem not with the full set of basis vectors, but with a subset of basis vectors. Let us consider an $m$-level basis graph, $m \ll N$, with its root at the current node of the search, termed the guiding graph, as this subset of basis vectors. The search process will move the guiding graph around through the $N$-level basis graph.

Intuition about the problem suggests that if the basis vector corresponding to true scattering behavior is not included in the guiding graph when the inverse problem is solved in a sparsity enforcing manner, then the resulting solution coefficient vector a will have a non-zero coefficient for the basis vector most 'similar' to the truth. In terms of the $N$-level basis graph, intuition suggests that if the true coefficient is far down in the basis graph, but the inverse problem is solved with only basis vectors from a guiding graph near the top of the $N$-level basis graph, then coefficients in the first $m-1$ levels will be zero and coefficients in level $m$ may be non-zero. In the same vein, if the guiding graph is rooted below the true coefficient, then the root coefficient may be non-zero and the coefficients in levels two through $m$ will be zero. Again, intuition suggests that if the guiding graph is such that it contains the true coefficient, then the true coefficient will be non-zero and the rest of the coefficients zero.

Before arriving at the search procedure and heuristics, let us first confirm the above intuition through experimentation for $N=400, m=8$, and implicitly $P=1$. The 400 angle samples are over the interval $\left[-55^{\circ},+55^{\circ}\right]$, the number of frequencies $K=3$ with values $7.047 \mathrm{GHz}, 7.059 \mathrm{GHz}$, and $7.070 \mathrm{GHz}$, and the regularization parameter $\alpha=150$. The 8-level guiding graph contains 36 nodes. In the first experiment, with results in Fig. 3, the guiding graph is fixed with root at the left-most node of level 200 in the basis graph. The true scattering behavior is varied from isotropic, to anisotropic with medium angular extent, to anisotropic with just one angle sample non-zero. In terms of the 400-level basis graph, the true coefficient is varied, starting at the root node, through all nodes along the left edge of the graph, to the left-most node of level 400, as diagrammed in the left portion of Fig. 3. The large triangle is the 400-level basis graph, the tiny filled triangle is the fixed guiding graph, and the arrows along the left edge indicate the variation of the true node. In the two plots, the angular extent of the true scattering behavior is plotted on the horizontal axis. In the top plot, the coefficient magnitudes for all 36 coefficients associated with the basis vectors in the guiding graph are plotted on the vertical axis, whereas in the bottom plot, coefficient magnitudes are indicated by shading (white is zero) and each horizontal strip is for each of the 36 different coefficients. The coefficient values are obtained by solving the inverse problem using the quasi-Newton method. Most coefficients are zero for all true scattering behaviors in this experiment. Lines on the plots are labeled in correspondence with node labels in Fig. 2. The figure shows that in agreement with intuition, in the regime where the guiding graph is below the true coefficient, the root node (node $h$ ) is non-zero. In the regime where the guiding graph covers the true coefficient, the correct node is non-zero. Also in agreement with intuition, when the guiding graph is above the true coefficient, the node in the last level closest to the truth (node $a$ ) is non-zero and others are zero. 

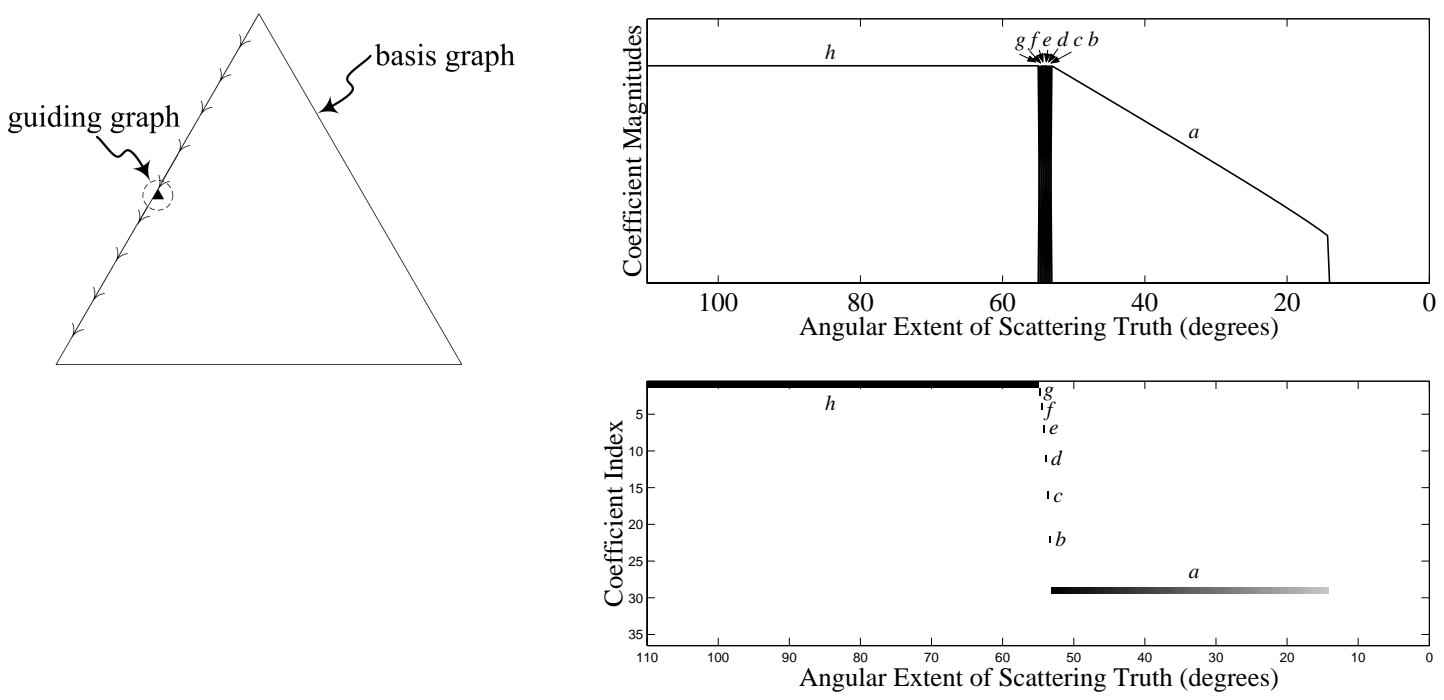

Figure 3. Coefficient magnitudes in $m$-level guiding graph as true scattering behavior is varied from isotropic to highly anisotropic. The $m$-level guiding graph is fixed with top node having angular extent $55.3^{\circ}$ and $m^{\text {th }}$ row nodes having angular extent $53.1^{\circ}$.
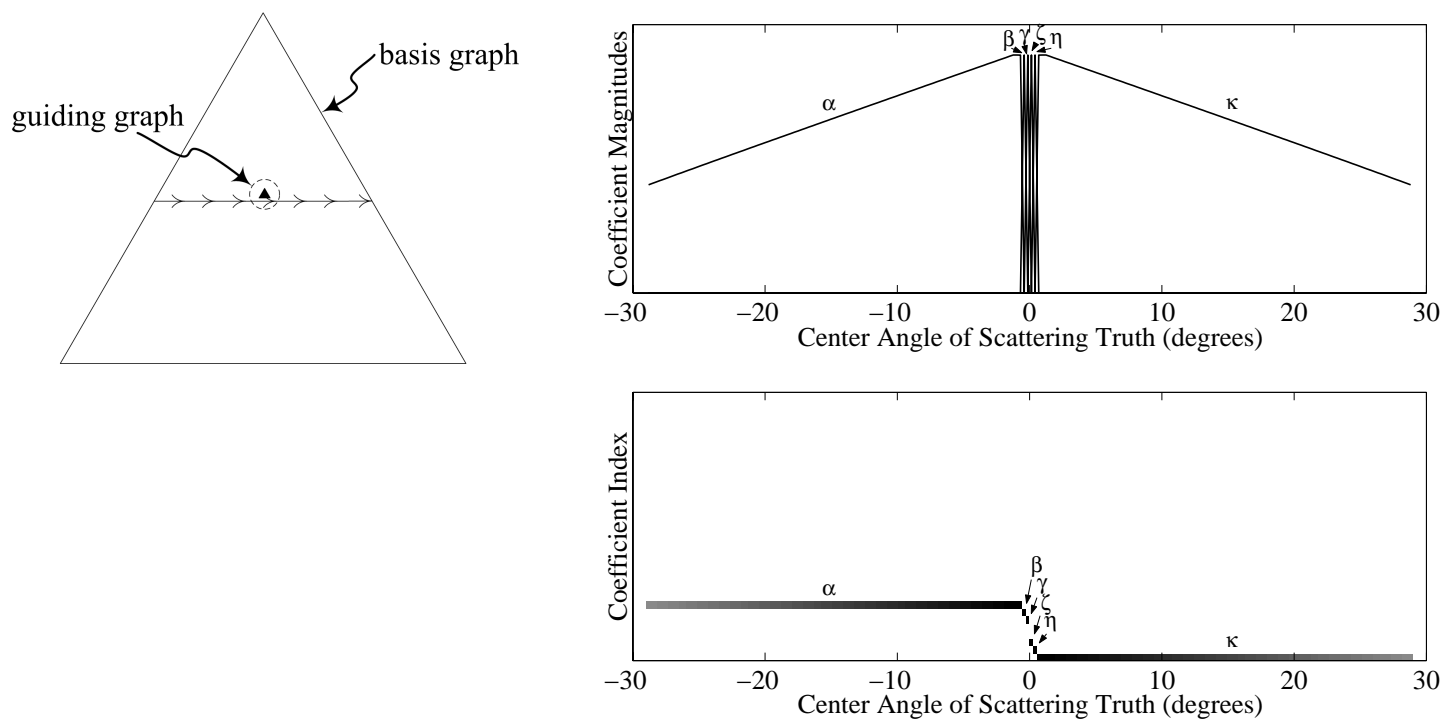

Figure 4. Coefficient magnitudes in $m$-level guiding graph as center angle of true scattering behavior is varied. The $m$-level guiding graph is fixed, covering center angles $\left[-1.0^{\circ},+1.0^{\circ}\right]$.

The experiment yielding the results of Fig. 4 has the same setup, but the guiding graph is fixed with root at the center node of level 200 instead of the left-most node. The true node is varied from left to right across the basis graph at level 210, three levels below the bottom of the guiding graph, effectively changing the center angle of the anisotropy, but leaving the extent constant. This figure is organized in the same manner as Fig. 3, but the horizontal axis features the center angle rather than angular extent. From these results, first it is apparent that only nodes in the last level of the guiding graph are non-zero, reconfirming results from the previous experiment. Second, it can be seen that when the truth is to the left of the guiding graph, the left-most node of the $m^{\text {th }}$ level (node $\alpha$ ) is non-zero. Similarly, when the truth is to the right, the right node (node $\kappa$ ) is non-zero; when the 
truth is underneath the 8-level graph, nodes along the last level (nodes $\beta-\eta$ ) are non-zero.

Intuition along with these experimental validations suggests simple stopping conditions and heuristics. One stopping criterion is to stop when all of the nodes in level $m$ (nodes $\alpha-\kappa$ ) are zero during the search. A heuristic for the search is also apparent based on the coefficient values of the $m$ nodes in level $m$. Due to the structure of the basis graph, each node has two children, so the heuristic will be used to determine whether the next guiding graph root will be the left child or the right child of the current guiding graph root node. Based on the second experiment, one reasonable idea is to take the weighted average of the coefficient magnitudes of the bottom level - the search can then be guided towards the side the average indicates to be stronger. The basis used in calculating the given heuristic and stopping criterion has $\mathcal{O}(1)$ columns for each spatial location and $\mathcal{O}(P)$ columns for $P$ spatial locations, providing savings in terms of memory.

For the case of a single spatial location, $P=1$, the algorithm is as follows. The inverse problem $\mathbf{r}=\boldsymbol{\Phi}^{(i)} \hat{\mathbf{a}}^{(i)}$ is solved for each iteration $i$ of the search. Then, $\hat{\mathbf{a}}^{(i)}$ is tested for the stopping condition. If the search is to continue, the heuristic is calculated to determine which one of two choices $\boldsymbol{\Phi}^{(i+1)}$ will be. The initial set of basis vectors $\boldsymbol{\Phi}^{(1)}$ is the set with the largest angular extent located in the top $m$ levels of the $N$-level basis graph. For the general case of multiple spatial locations, $P$ searches are performed simultaneously, but not independently. As in the single position case, $\mathbf{r}=\boldsymbol{\Phi}^{(i)} \hat{\mathbf{a}}^{(i)}$ is still solved on each iteration, but now individual block matrices $\boldsymbol{\Phi}_{p}^{(i)}$ evolve based on their corresponding $\hat{\mathbf{a}}_{p}^{(i)}$. For example, the first spatial location's coefficients $\hat{\mathbf{a}}_{1}^{(i)}$ may satisfy the stopping condition, in which case $\boldsymbol{\Phi}_{1}^{(i+1)}=\boldsymbol{\Phi}_{1}^{(i)}$. The second spatial location's coefficients may indicate through the heuristic that the search should proceed to the left child, so $\boldsymbol{\Phi}_{2}^{(i)}$ is updated accordingly, and so on. The overall search terminates when all of the $\hat{\mathbf{a}}_{p}^{(i)}$ satisfy the stopping criterion. The $P$ searches are coupled because the inverse problem is solved jointly for all spatial locations on every iteration. When there are multiple spatial locations, contributions from different positions interact. As stated, the algorithm allows for contributions from more than one basis vector per spatial location in the final solution, but those basis vectors must be within the span of a guiding graph. The guiding graph may be enlarged to allow for contributions from disparate basis vectors at additional expense, the extreme being to take the guiding graph as the full basis graph.

A number of variations to the basic algorithm presented above may be made that further reduce memory or computation. First, the back-tracking component of the algorithm may be removed; if the search terminates before reaching a leaf, then this does not change anything. Without back-tracking, the search becomes greedier and takes $\mathcal{O}(N)$ iterations, whereas with back-tracking there are $\mathcal{O}\left(N^{2}\right)$ iterations. The guiding graph need not be an $m$-level basis graph; for example, the graph may be thinned and include the top level, bottom level, and a few intermediate levels rather than all intermediate levels. A further approximation can be introduced into the search without back-tracking to reduce the average-case dependence of the number of total basis vectors on $P$. We can fix the contribution from a spatial location after its coefficients have been found. In the algorithm, this implies that once the stopping criterion is met and maintained for a few iterations at position $p$, the observation data $\mathbf{r}$ is updated to be $\mathbf{r}^{\prime}=\mathbf{r}-\boldsymbol{\Phi}_{p} \mathbf{a}_{p}$, and $\boldsymbol{\Phi}_{p}$ is removed from matrix $\boldsymbol{\Phi}$, thereby reducing the number of columns in $\boldsymbol{\Phi}$. This list of variations is far from exhaustive. The next section gives examples of using our formulation for anisotropy characterization.

\section{EXAMPLES}

In this section, we present three examples of anisotropy characterization; the first uses the quasi-Newton method on a scene with XPatch data of canonical point scattering, the second uses the graph-structured algorithm on synthetic data, and the last uses the graph-structured algorithm on the backhoe dataset. The first two examples are mainly for illustrative purposes.

In this first example, there are four spatial locations (pixels) at $(0,0),\left(0, \frac{1}{2}\right),\left(\frac{1}{2}, 0\right)$, and $\left(\frac{1}{2}, \frac{1}{2}\right)$ meters. We use measurements at $K=3$ frequencies $9 \mathrm{GHz}, 9.016 \mathrm{GHz}$, and $9.032 \mathrm{GHz}$ over the $N=50$ angle samples equally spaced over a $98^{\circ}$ aperture. Illustrating the fact that all spatial locations need not contain point scattering centers, in this example, two of the spatial locations have no scatterers. The scattering centers at the other two spatial locations exhibit realistic, i.e. from XPatch predictions, aspect-dependent scattering behavior. We solve $\mathbf{r}=\boldsymbol{\Phi} \mathbf{a}$ using the pseudo-inverse to obtain the least-squares solution as a baseline for comparison. We also use 

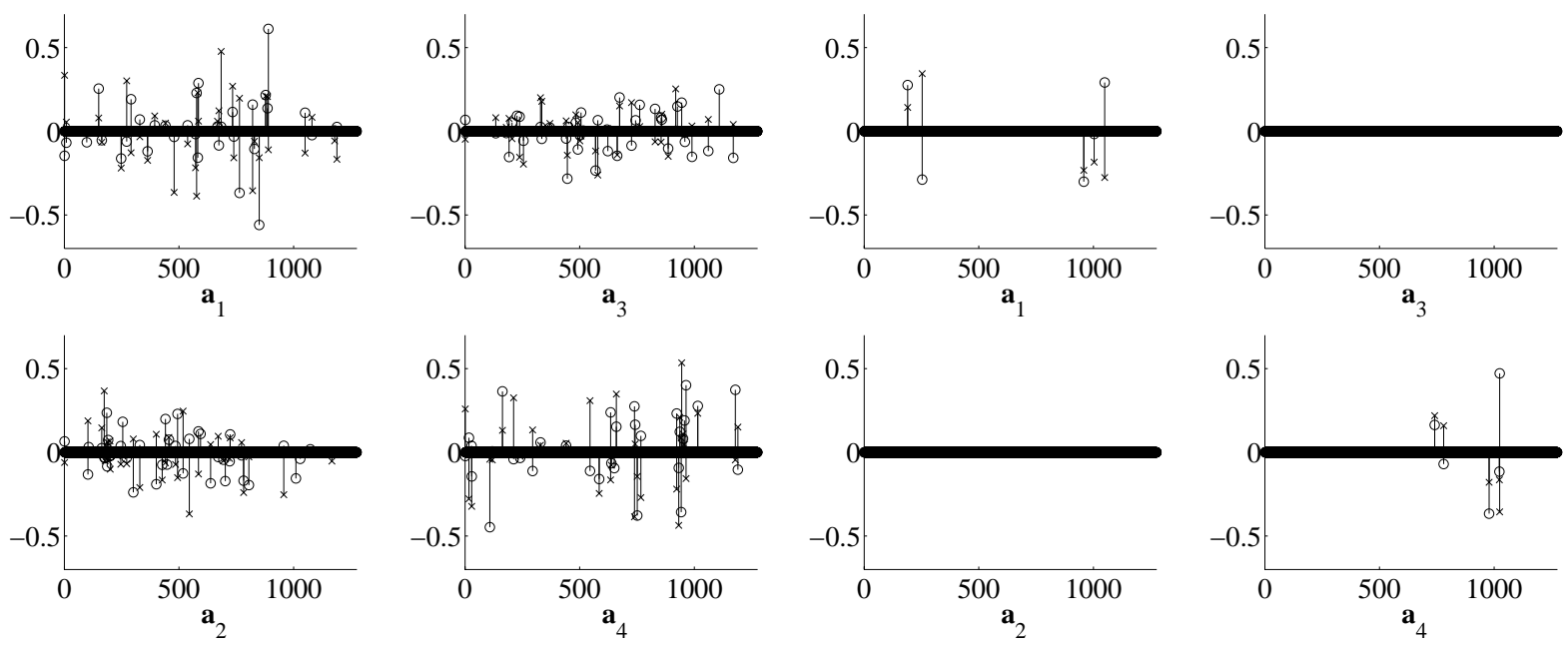

(a)
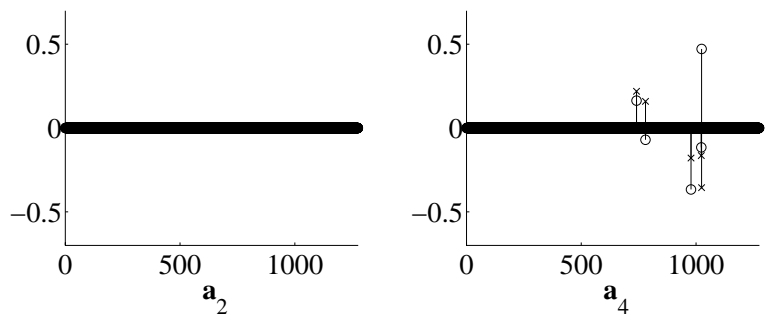

(b)

Figure 5. Coefficient vectors a from (a) least-squares solution and (b) sparse solution, with real part $\circ$ and imaginary part $\times$.

the quasi-Newton method with the $\ell_{k}$-norm having $k=0.1$ and the regularization parameter $\alpha=1$ to obtain a solution.

Fig. 5 gives stem plots that show the values of the coefficients in the solution a vectors, the least-squares solution on the left and the solution with regularization on the right. The stems topped by $\circ$ give the value of the real part and the stems topped by $\times$, the imaginary part. For $N=50$, there are $M=1275$ basis vectors per spatial location; the subplots enumerate the corresponding 1275 coefficients from left to right as in Fig. 1. As expected, the a vector on the right is much sparser due to the $\ell_{0.1}$ regularization term. In fact, the coefficients corresponding to spatial locations without scattering centers are all nearly zero in the sparse solution, whereas the least-squares solution has many large-valued coefficients.

Now let us inspect what these coefficients map to in terms of estimated $s\left(x_{p}, y_{p}, \theta\right)$ functions. Fig. 6 shows the magnitude of the solutions in blue overlaid on the underlying truth in black. The sparse solution is more accurate in its representation of the underlying truth than the least-squares solution, a consequence of the fact that basis vectors of contiguous anisotropy are fairly good at sparsely representing realistic aspect-dependence. It should be noted that the least-squares solution perfectly matches the measurement vector r, whereas the sparse solution does not, but data fit is not our primary concern. It should also be noted that if we were to perform image formation without anisotropy characterization, we would have four likely inaccurate pixel values, rather than four accurate functions of $\theta$.

The first example illustrated the importance of sparsity and gave an indication that contiguous basis vectors are a reasonable choice. The second example, with 7 scattering centers and $N=1541$ angles, shows the operation of the graph-structured algorithm using synthetic data. The aperture is from $-10^{\circ}$ to $+100^{\circ}$ and the scattering centers have anisotropy of varying angular extents with a raised triangle pulse shape. Note that in this example, we use raised triangle pulse shapes for the $\mathbf{b}_{m}$ vectors as well. As a preprocessing step, we first locate the scattering centers by peak extraction on a conventionally formed image. In this example, the extracted scatterer locations are within $4 \mathrm{~mm}$ of the truth, with the main source of error being the discrete grid of pixels in the conventionally-formed image. The conventional image and the extracted scattering center locations are shown in Fig. 7c. Then with $P=7$ and using measurements at the $K=3$ frequencies $7.047 \mathrm{GHz}, 7.059 \mathrm{GHz}$, and 7.070 $\mathrm{GHz}$, we run the graph-structured algorithm without back-tracking and with the search heuristic and stopping condition discussed in Sec. 3. The guiding graph has 16 levels.

The simultaneous searches are shown in Fig. 7a, where the 1541-level basis graph is indicated by the triangular outline. The resulting $s\left(x_{p}, y_{p}, \theta\right)$ estimate magnitudes are shown in Fig. $7 \mathrm{~b}$ as blue lines overlaid on the black 

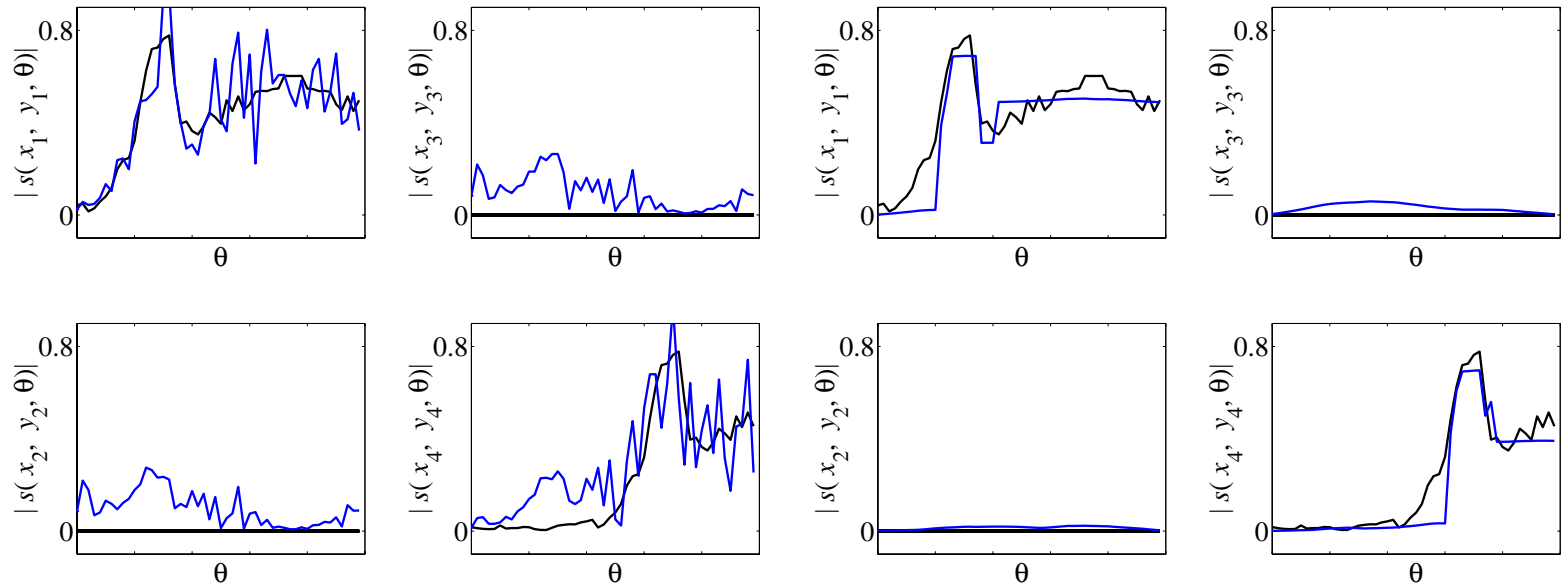

(a)
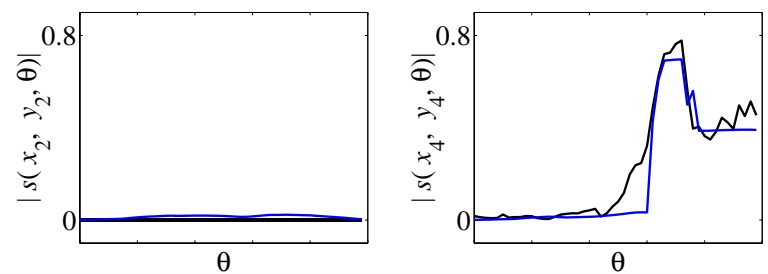

(b)

Figure 6. Magnitude of characterized anisotropy from (a) least-squares solution and (b) sparse solution, plotted in blue overlaid on truth.

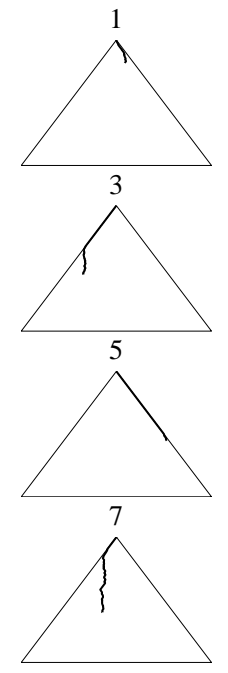

(a)
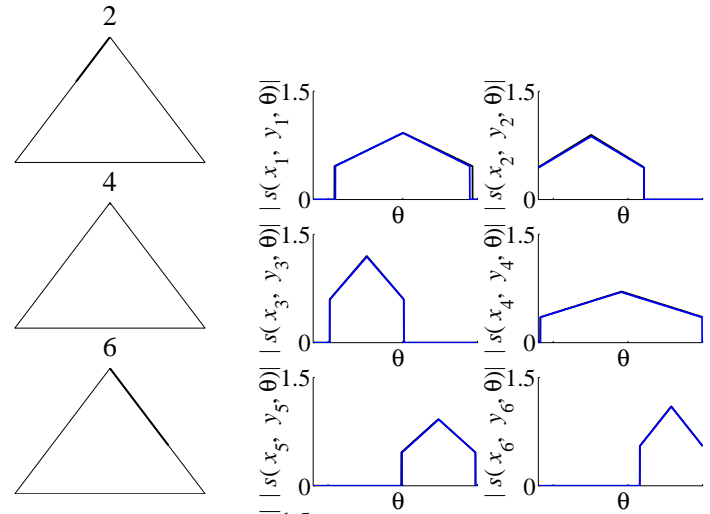

$\overline{\bar{\theta}} 1.5$

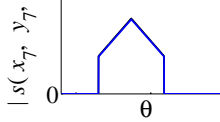

(b)

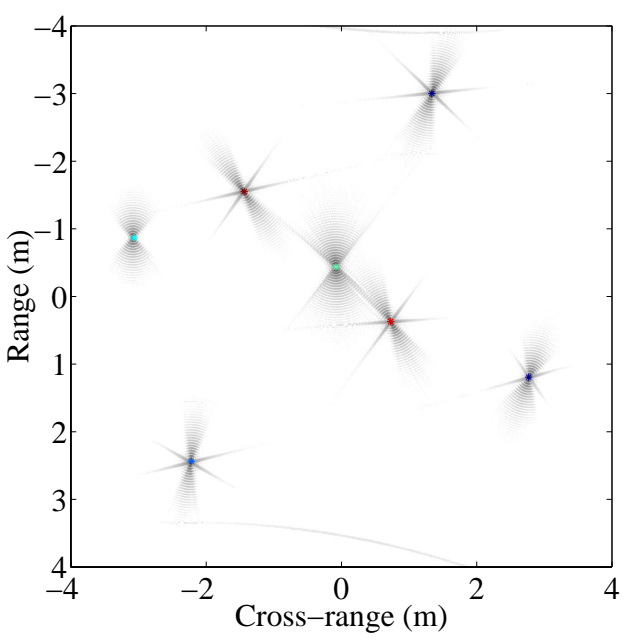

(c)

Figure 7. Results of example using graph-structured algorithm: (a) search paths, (b) solution $s\left(x_{p}, y_{p}, \theta\right)$ overlaid on truth, and (c) color-coded anisotropy center. 


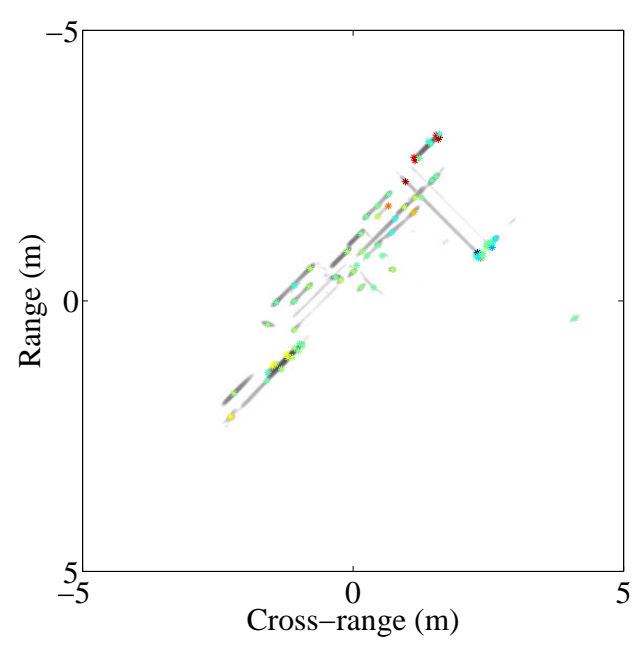

(a)

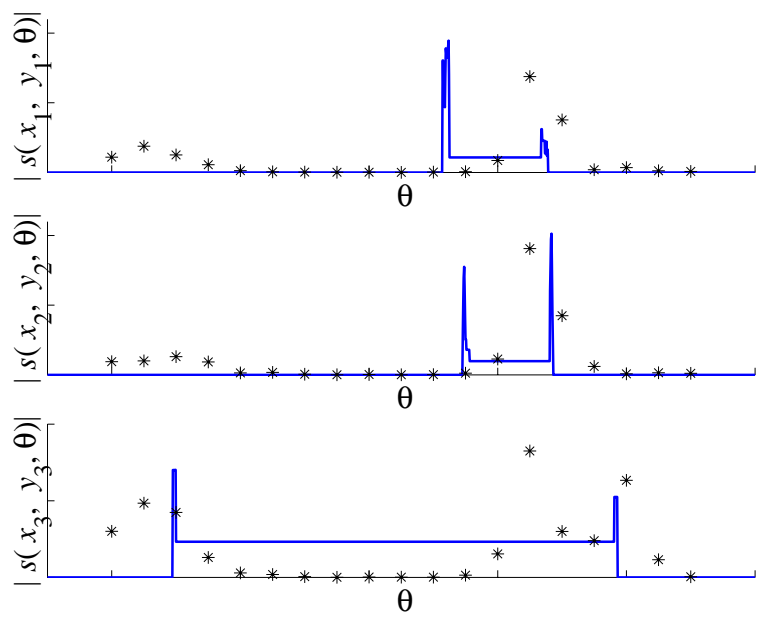

(b)

Figure 8. Results of backhoe example using graph-structured algorithm: (a) color-coded anisotropy center, and (b) sample characterized anisotropies.

truth. The estimates are nearly indistinguishable from the true anisotropies. The center of the anisotropy is indicated in Fig. 7c as a color, where dark red corresponds to the center angle closest to $-10^{\circ}$ and blue corresponds to the center angle closest to $100^{\circ}$, with the colors cycling red to green to blue. The results are accurate, but moreover, the search paths are fairly direct and would not require back-tracking even if it were available. We see that the formulation is not restricted to rectangle-shaped basis vectors - other pulse shapes may be used as well, as long as they can be used to sparsely represent plausible anisotropy.

The final example, with a dataset from the Backhoe Data Dome, ${ }^{15}$ uses the same algorithm as the previous example. The data also has $N=1541$ angle samples over an aperture from $-10^{\circ}$ to $+100^{\circ}$. $P=75$ spatial locations are extracted from a composite image of conventionally-formed subaperture images ${ }^{11}$ and then the greedy graph-structured algorithm is applied to the data with $K=3$ and frequencies $7.047 \mathrm{GHz}, 9.994 \mathrm{GHz}$, and $12.953 \mathrm{GHz}$. The solution is displayed in Fig. 8a, also color-coded. The characterized anisotropies of a few scattering centers are also shown in Fig. 8. The solid line indicates our solution, whereas the asterisks show subaperture pixel values from conventional imaging with overlapping $20^{\circ}$ subapertures. ${ }^{11}$ The vertical axes for the line and the set of asterisks are scaled differently to allow comparison. For the first two scattering centers, the subaperture pixel values indicate contiguous extents of anisotropy and our algorithm also detects strong responses at those angles. However, the type of solution we are able to produce is more detailed in $\theta$, especially because the results indicate that anisotropy persistence is not matched to subaperture width. In the third scattering center, the subaperture pixel values indicate two disjoint segments of anisotropy. However, the greedy algorithm may only use basis vectors that lie within a guiding graph to explain the anisotropy. Nevertheless, our algorithm does the best it can to produce two peaks via a positive-valued guiding graph root coefficient and negative-valued leaf coefficient. Multiple candidate search is an approach that would allow for better performance in such instances.

\section{CONCLUSION}

We have presented a novel approach to SAR image formation. The methodology is general in that it can be applied to a wide variety of overcomplete basis representations. Here we have focused on its utility in describing anisotropic scattering behavior of complex reflections in wide-angle SAR data. The primary advantage of the approach derives from a convenient organization of the basis vectors. The structure allows for a computationally efficient search for the solution of a large sparse regularized inverse problem by evaluating a subset of basis vectors at each iteration. The method demonstrated excellent results on synthetic data, but more importantly, characterized anisotropy to a level of fine detail not possible with subaperture analysis on complex scenes such as 
the backhoe dataset. Future work will consider extending the formulation to incorporate another issue that arises in wide-angle imaging, i.e. that certain scattering mechanisms appear to move or migrate in spatial location as a function of aspect angle. Also, we may consider extending the cost function (2) to include preferences other than just sparsity among basis coefficients.

\section{ACKNOWLEDGMENTS}

The authors would like to thank Rajan Bhalla for providing XPatch data and Randy Moses for fruitful exchange. This work was partially supported by the Air Force Research Laboratory under Grant FA8650-04-1-1719, and Grant FA8650-04-C-1703 (through subcontract 04079-6918 from BAE Systems Advanced Information Technologies). The first author is also supported by the National Science Foundation Graduate Research Fellowship Program.

\section{REFERENCES}

1. L. C. Potter and R. L. Moses, "Attributed scattering centers for SAR ATR," IEEE Trans. Image Processing 6, pp. 79-91, Jan. 1997.

2. L. C. Trintinalia, R. Bhalla, and H. Ling, "Scattering center parameterization of wide-angle backscattered data using adaptive Gaussian representation," IEEE Trans. Antennas Propagat. 45, pp. 1664-1668, Nov. 1997.

3. M. J. Gerry, L. C. Potter, I. J. Gupta, and A. van der Merwe, "A parametric model for synthetic aperture radar measurements," IEEE Trans. Antennas Propagat. 47, pp. 1179-1188, July 1999.

4. A. Genell and D. Lösaus, "On the Cramer Rao bound in determining scattering center parameters using high resolution radar," Master's thesis, Chalmers University of Technology and Göteborg University, Göteborg, Sweden, 2001.

5. M. R. Allen and L. E. Hoff, "Wide-angle wideband SAR matched filter image formation for enhanced detection performance," in SPIE Symposium, Algorithms for Synthetic Aperture Radar Imagery, D. A. Giglio, ed., (Orlando, Florida), Apr. 1994.

6. L. R. Flake, S. C. Ahalt, and A. K. Krishnamurthy, "Detecting anisotropic scattering with hidden Markov models," IEE Proceedings - Radar, Sonar $E 3$ Navigation 144, pp. 81-86, Apr. 1997.

7. P. Runkle, L. H. Nguyen, J. H. McClellan, and L. Carin, "Multi-aspect target detection for SAR imagery using hidden Markov models," IEEE Trans. Geosci. Remote Sensing 39, pp. 46-55, Jan. 2001.

8. B. Krishnapuram, J. Sichina, and L. Carin, "Physics-based detection of targets in SAR imagery using support vector machines," IEEE Sensors J. 3, pp. 147-157, Apr. 2003.

9. L. Ferro-Famil, A. Reigber, E. Pottier, and W.-M. Boerner, "Scene characterization using subaperture polarimetric SAR data," IEEE Trans. Geosci. Remote Sensing 41, pp. 2264-2276, Oct. 2003.

10. R. L. Moses, L. C. Potter, and M. Çetin, "Wide angle SAR imaging," in SPIE Defense and Security Symposium, Algorithms for Synthetic Aperture Radar Imagery XI, E. G. Zelnio and F. D. Garber, eds., (Orlando, Florida), Apr. 2004.

11. M. Çetin and R. L. Moses, "SAR imaging from partial-aperture data with frequency-band omissions," in SPIE Defense and Security Symposium, Algorithms for Synthetic Aperture Radar Imagery XII, E. G. Zelnio and F. D. Garber, eds., (Orlando, Florida), Mar. 2005.

12. A. J. Kim, J. W. Fisher, III, and A. S. Willsky, "Detection and analysis of anisotropic scattering in SAR data," Multidimensional Systems and Signal Processing 14, pp. 49-82, Jan. 2003.

13. D. M. Malioutov, "A sparse signal reconstruction perspective for source localization with sensor arrays," Master's thesis, Massachusetts Institute of Technology, Cambridge, Massachusetts, 2003.

14. M. Çetin and W. C. Karl, "Feature-enhanced synthetic aperture radar image formation based on nonquadratic regularization," IEEE Trans. Image Processing 10, pp. 623-631, Apr. 1997.

15. "Backhoe data dome and Visual-D challenge problem." Available at Air Force Research Laboratory Sensor Data Management System (https://www.sdms.afrl.af.mil/main.php), 2004. 